\title{
Measurement of the top quark mass using the invariant mass of lepton pairs in soft muon $b$-tagged events
}

T. Aaltonen, ${ }^{24}$ J. Adelman, ${ }^{14}$ T. Akimoto, ${ }^{56}$ B. Álvarez González, ${ }^{12, u}$ S. Amerio, ${ }^{44 b, 44 a}$ D. Amidei ${ }^{35}$ A. Anastassov, ${ }^{39}$ A. Annovi ${ }^{20}$ J. Antos, ${ }^{15}$ G. Apollinari, ${ }^{18}$ A. Apresyan, ${ }^{49}$ T. Arisawa,${ }^{58}$ A. Artikov,${ }^{16}$ W. Ashmanskas, ${ }^{18}$ A. Attal,${ }^{4}$ A. Aurisano, ${ }^{54}$ F. Azfar, ${ }^{43}$ W. Badgett, ${ }^{18}$ A. Barbaro-Galtieri, ${ }^{29}$ V. E. Barnes ${ }^{49}$ B. A. Barnett, ${ }^{26}$ P. Barria,${ }^{47 c, 47 a}$ P. Bartos, ${ }^{15}$ V. Bartsch, ${ }^{31}$ G. Bauer, ${ }^{33}$ P.-H. Beauchemin, ${ }^{34}$ F. Bedeschi, ${ }^{47 a}$ D. Beecher,${ }^{31}$ S. Behari, ${ }^{26}$ G. Bellettini, ${ }^{47 b, 47 a}$ J. Bellinger, ${ }^{60}$ D. Benjamin, ${ }^{17}$ A. Beretvas, ${ }^{18}$ J. Beringer, ${ }^{29}$ A. Bhatti, ${ }^{51}$ M. Binkley, ${ }^{18}$ D. Bisello, ${ }^{44 b, 44 a}$ I. Bizjak, ${ }^{31, z}$ R. E. Blair, ${ }^{2}$ C. Blocker, ${ }^{7}$ B. Blumenfeld ${ }^{26}$ A. Bocci, ${ }^{17}$ A. Bodek,${ }^{50}$ V. Boisvert ${ }^{50}$ G. Bolla, ${ }^{49}$ D. Bortoletto, ${ }^{49}$ J. Boudreau ${ }^{48}$ A. Boveia, ${ }^{11}$ B. Brau, ${ }^{11, b}$ A. Bridgeman,${ }^{25}$ L. Brigliadori, ${ }^{6 b, 6 a}$ C. Bromberg,${ }^{36}$ E. Brubaker,${ }^{14}$ J. Budagov, ${ }^{16}$ H. S. Budd, ${ }^{50}$

S. Budd, ${ }^{25}$ S. Burke, ${ }^{18}$ K. Burkett, ${ }^{18}$ G. Busetto, ${ }^{44 b, 44 a}$ P. Bussey, ${ }^{22}$ A. Buzatu, ${ }^{34}$ K. L. Byrum, ${ }^{2}$ S. Cabrera, ${ }^{17, w}$ C. Calancha, ${ }^{32}$ M. Campanelli, ${ }^{36}$ M. Campbell, ${ }^{35}$ F. Canelli, ${ }^{14,18}$ A. Canepa,${ }^{46}$ B. Carls, ${ }^{25}$ D. Carlsmith,${ }^{60}$ R. Carosi,${ }^{47 a}$ S. Carrillo, ${ }^{19,0}$ S. Carron,${ }^{34}$ B. Casal, ${ }^{12}$ M. Casarsa, ${ }^{18}$ A. Castro, ${ }^{6 b, 6 a}$ P. Catastini,${ }^{47 c, 47 a}$ D. Cauz,${ }^{55 b, 55 a}$ V. Cavaliere, ${ }^{47 c, 47 a}$ M. Cavalli-Sforza, ${ }^{4}$ A. Cerri, ${ }^{29}$ L. Cerrito, ${ }^{31, q}$ S. H. Chang, ${ }^{28}$ Y. C. Chen, ${ }^{1}$ M. Chertok,${ }^{8}$ G. Chiarelli, ${ }^{47 a}$ G. Chlachidze, ${ }^{18}$ F. Chlebana, ${ }^{18}$ K. Cho, ${ }^{28}$ D. Chokheli, ${ }^{16}$ J. P. Chou, ${ }^{23}$ G. Choudalakis, ${ }^{33}$ S. H. Chuang, ${ }^{53}$ K. Chung, ${ }^{18, p}$ W. H. Chung, ${ }^{60}$ Y. S. Chung, ${ }^{50}$ T. Chwalek, ${ }^{27}$ C. I. Ciobanu, ${ }^{45}$ M. A. Ciocci, ${ }^{47 c, 47 a}$ A. Clark, ${ }^{21}$ D. Clark, ${ }^{7}$ G. Compostella, ${ }^{44 a}$ M. E. Convery, ${ }^{18}$ J. Conway, ${ }^{8}$ M. Cordelli, ${ }^{20}$ G. Cortiana, ${ }^{44 b, 44 a}$ C. A. Cox,${ }^{8}$ D. J. Cox,${ }^{8}$ F. Crescioli, ${ }^{47 b, 47 a}$

C. Cuenca Almenar, ${ }^{8, w}$ J. Cuevas, ${ }^{12, \mathrm{u}}$ R. Culbertson, ${ }^{18}$ J. C. Cully, ${ }^{35}$ D. Dagenhart, ${ }^{18}$ M. Datta, ${ }^{18}$ T. Davies, ${ }^{22}$ P. de Barbaro, ${ }^{50}$ S. De Cecco, ${ }^{52 a}$ A. Deisher ${ }^{29}$ G. De Lorenzo, ${ }^{4}$ M. Dell'Orso,${ }^{47 b, 47 a}$ C. Deluca, ${ }^{4}$ L. Demortier,${ }^{51}$ J. Deng, ${ }^{17}$ M. Deninno, ${ }^{6 a}$ P. F. Derwent, ${ }^{18}$ A. Di Canto, ${ }^{47 b, 47 a}$ G. P. di Giovanni, ${ }^{45}$ C. Dionisi, ${ }^{52 b, 52 a}$ B. Di Ruzza, ${ }^{55 b, 55 a}$

J. R. Dittmann, ${ }^{5}$ M. D’Onofrio, ${ }^{4}$ S. Donati, ${ }^{47 b, 47 a}$ P. Dong, ${ }^{9}$ J. Donini,${ }^{44 a}$ T. Dorigo, ${ }^{44 a}$ S. Dube, ${ }^{53}$ J. Efron,${ }^{40}$ A. Elagin, ${ }^{54}$

R. Erbacher, ${ }^{8}$ D. Errede, ${ }^{25}$ S. Errede, ${ }^{25}$ R. Eusebi,${ }^{18}$ H. C. Fang, ${ }^{29}$ S. Farrington, ${ }^{43}$ W. T. Fedorko, ${ }^{14}$ R. G. Feild, ${ }^{61}$

M. Feindt, ${ }^{27}$ J. P. Fernandez, ${ }^{32}$ C. Ferrazza, ${ }^{47 d, 47 a}$ R. Field, ${ }^{19}$ G. Flanagan, ${ }^{49}$ R. Forrest, ${ }^{8}$ M. J. Frank, ${ }^{5}$ M. Franklin, ${ }^{23}$

J. C. Freeman, ${ }^{18}$ I. Furic, ${ }^{19}$ M. Gallinaro, ${ }^{52 a}$ J. Galyardt, ${ }^{13}$ J. E. Garcia, ${ }^{21}$ A. F. Garfinkel, ${ }^{49}$ P. Garosi,,${ }^{47,47 a}$ K. Genser, ${ }^{18}$ H. Gerberich, ${ }^{25}$ D. Gerdes, ${ }^{35}$ A. Gessler, ${ }^{27}$ S. Giagu, ${ }^{52 b, 52 a}$ V. Giakoumopoulou, ${ }^{3}$ P. Giannetti, ${ }^{47 a}$ K. Gibson, ${ }^{48}$ J. L. Gimmell, ${ }^{50}$ C. M. Ginsburg, ${ }^{18}$ N. Giokaris, ${ }^{3}$ M. Giordani, ${ }^{55 b, 55 a}$ P. Giromini, ${ }^{20}$ M. Giunta, ${ }^{47 a}$ G. Giurgiu, ${ }^{26}$ V. Glagolev, ${ }^{16}$ D. Glenzinski, ${ }^{18}$ M. Gold ${ }^{38}$ N. Goldschmidt,${ }^{19}$ A. Golossanov, ${ }^{18}$ G. Gomez, ${ }^{12}$ G. Gomez-Ceballos,${ }^{33}$ M. Goncharov, ${ }^{33}$ O. González, ${ }^{32}$ I. Gorelov, ${ }^{38}$ A. T. Goshaw, ${ }^{17}$ K. Goulianos,${ }^{51}$ A. Gresele, ${ }^{44 b, 44 a}$ S. Grinstein, ${ }^{23}$ C. Grosso-Pilcher, ${ }^{14}$ R. C. Group, ${ }^{18}$ U. Grundler, ${ }^{25}$ J. Guimaraes da Costa, ${ }^{23}$ Z. Gunay-Unalan, ${ }^{36}$ C. Haber ${ }^{29}$ K. Hahn, ${ }^{33}$ S. R. Hahn, ${ }^{18}$ E. Halkiadakis, ${ }^{53}$ B.-Y. Han,${ }^{50}$ J. Y. Han ${ }^{50}$ F. Happacher,${ }^{20}$ K. Hara,${ }^{56}$ D. Hare,${ }^{53}$ M. Hare,${ }^{57}$ S. Harper, ${ }^{43}$ R. F. Harr ${ }^{59}$ R. M. Harris, ${ }^{18}$ M. Hartz,${ }^{48}$ K. Hatakeyama, ${ }^{51}$ C. Hays,${ }^{43}$ M. Heck,${ }^{27}$ A. Heijboer, ${ }^{46}$ J. Heinrich, ${ }^{46}$ C. Henderson, ${ }^{33}$ M. Herndon, ${ }^{60}$ J. Heuser ${ }^{27}$ S. Hewamanage,${ }^{5}$ D. Hidas,${ }^{17}$ C. S. Hill, ${ }^{11, d}$ D. Hirschbuehl, ${ }^{27}$ A. Hocker, ${ }^{18}$ S. Hou, ${ }^{1}$ M. Houlden, ${ }^{30}$ S.-C. Hsu, ${ }^{29}$ B. T. Huffman, ${ }^{43}$ R. E. Hughes, ${ }^{40}$ U. Husemann, ${ }^{61}$ M. Hussein, ${ }^{36}$ J. Huston, ${ }^{36}$ J. Incandela, ${ }^{11}$ G. Introzzi ${ }^{47 a}$ M. Iori,${ }^{52 b, 52 a}$ A. Ivanov, ${ }^{8}$ E. James, ${ }^{18}$ D. Jang, ${ }^{13}$ B. Jayatilaka, ${ }^{17}$ E. J. Jeon, ${ }^{28}$ M. K. Jha, ${ }^{6 a}$ S. Jindariani, ${ }^{18}$ W. Johnson, ${ }^{8}$ M. Jones,${ }^{49}$ K. K. Joo, ${ }^{28}$ S. Y. Jun, ${ }^{13}$ J. E. Jung, ${ }^{28}$ T. R. Junk, ${ }^{18}$ T. Kamon,${ }^{54}$ D. Kar, ${ }^{19}$ P. E. Karchin ${ }^{59}$ Y. Kato, ${ }^{42, n}$ R. Kephart, ${ }^{18}$ W. Ketchum, ${ }^{14}$ J. Keung, ${ }^{46}$ V. Khotilovich, ${ }^{54}$ B. Kilminster, ${ }^{18}$ D. H. Kim, ${ }^{28}$ H. S. Kim, ${ }^{28}$ H. W. Kim, ${ }^{28}$ J.E. Kim, ${ }^{28}$ M. J. Kim, ${ }^{20}$ S. B. Kim, ${ }^{28}$ S. H. Kim,${ }^{56}$ Y. K. Kim, ${ }^{14}$ N. Kimura, ${ }^{56}$ L. Kirsch, ${ }^{7}$ S. Klimenko, ${ }^{19}$ B. Knuteson, ${ }^{33}$ B. R. Ko, ${ }^{17}$ K. Kondo, ${ }^{58}$ D. J. Kong, ${ }^{28}$ J. Konigsberg, ${ }^{19}$ A. Korytov, ${ }^{19}$ A. V. Kotwal, ${ }^{17}$ M. Kreps,${ }^{27}$ J. Kroll, ${ }^{46}$ D. Krop,${ }^{14}$ N. Krumnack,,${ }^{5}$ M. Kruse, ${ }^{17}$ V. Krutelyov,${ }^{11}$ T. Kubo, ${ }^{56}$ T. Kuhr, ${ }^{27}$ N. P. Kulkarni, ${ }^{59}$ M. Kurata, ${ }^{56}$ S. Kwang, ${ }^{14}$ A. T. Laasanen ${ }^{49}$ S. Lami, ${ }^{47 a}$ S. Lammel, ${ }^{18}$ M. Lancaster, ${ }^{31}$ R. L. Lander, ${ }^{8}$ K. Lannon ${ }^{40, t}$

A. Lath ${ }^{53}$ G. Latino, ${ }^{47 c, 47 a}$ I. Lazzizzera, ${ }^{44 b, 44 a}$ T. LeCompte, ${ }^{2}$ E. Lee, ${ }^{54}$ H. S. Lee, ${ }^{14}$ S. W. Lee, ${ }^{54, v}$ S. Leone,${ }^{47 a}$

J. D. Lewis,${ }^{18}$ C.-S. Lin, ${ }^{29}$ J. Linacre, ${ }^{43}$ M. Lindgren, ${ }^{18}$ E. Lipeles,${ }^{46}$ T. M. Liss,${ }^{25}$ A. Lister, ${ }^{8}$ D. O. Litvintsev, ${ }^{18}$ C. Liu,${ }^{48}$

T. Liu, ${ }^{18}$ N. S. Lockyer ${ }^{46}$ A. Loginov, ${ }^{61}$ M. Loreti, ${ }^{44 b, 44 a}$ L. Lovas, ${ }^{15}$ D. Lucchesi, ${ }^{44 b, 44 a}$ C. Luci, ${ }^{52 b, 52 a}$ J. Lueck, ${ }^{27}$

P. Lujan, ${ }^{29}$ P. Lukens, ${ }^{18}$ G. Lungu,${ }^{51}$ L. Lyons,${ }^{43}$ J. Lys,${ }^{29}$ R. Lysak,${ }^{15}$ D. MacQueen, ${ }^{34}$ R. Madrak, ${ }^{18}$ K. Maeshima, ${ }^{18}$

K. Makhoul, ${ }^{33}$ T. Maki, ${ }^{24}$ P. Maksimovic, ${ }^{26}$ S. Malde, ${ }^{43}$ S. Malik,${ }^{31}$ G. Manca, ${ }^{30, f}$ A. Manousakis-Katsikakis,${ }^{3}$

F. Margaroli, ${ }^{49}$ C. Marino, ${ }^{27}$ C. P. Marino, ${ }^{25}$ A. Martin, ${ }^{61}$ V. Martin, ${ }^{22,1}$ M. Martínez,${ }^{4}$ R. Martínez-Ballarín, ${ }^{32}$

T. Maruyama, ${ }^{56}$ P. Mastrandrea, ${ }^{52 a}$ T. Masubuchi, ${ }^{56}$ M. Mathis, ${ }^{26}$ M. E. Mattson, ${ }^{59}$ P. Mazzanti, ${ }^{6 a}$ K. S. McFarland, ${ }^{50}$

P. McIntyre ${ }^{54}$ R. McNulty, ${ }^{30, k}$ A. Mehta, ${ }^{30}$ P. Mehtala, ${ }^{24}$ A. Menzione,${ }^{47 a}$ P. Merkel,${ }^{49}$ C. Mesropian,${ }^{51}$ T. Miao, ${ }^{18}$ N. Miladinovic, ${ }^{7}$ R. Miller, ${ }^{36}$ C. Mills,${ }^{23}$ M. Milnik, ${ }^{27}$ A. Mitra, ${ }^{1}$ G. Mitselmakher,${ }^{19}$ H. Miyake, ${ }^{56}$ S. Moed,${ }^{23}$ N. Moggi, ${ }^{6 a}$ M. N. Mondragon, ${ }^{18, o}$ C. S. Moon, ${ }^{28}$ R. Moore, ${ }^{18}$ M. J. Morello, ${ }^{47 a}$ J. Morlock,${ }^{27}$ P. Movilla Fernandez,${ }^{18}$

J. Mülmenstädt, ${ }^{29}$ A. Mukherjee, ${ }^{18}$ Th. Muller, ${ }^{27}$ R. Mumford, ${ }^{26}$ P. Murat, ${ }^{18}$ M. Mussini, ${ }^{6 b, 6 a}$ J. Nachtman, ${ }^{18, p}$ Y. Nagai, ${ }^{56}$ 
A. Nagano,${ }^{56}$ J. Naganoma,${ }^{56}$ K. Nakamura,${ }^{56}$ I. Nakano, ${ }^{41}$ A. Napier,${ }^{57}$ V. Necula,${ }^{17}$ J. Nett ${ }^{60}$ C. Neu, ${ }^{46, x}$ M. S. Neubauer, ${ }^{25}$ S. Neubauer,${ }^{27}$ J. Nielsen,${ }^{29, \mathrm{~h}}$ L. Nodulman,${ }^{2}$ M. Norman, ${ }^{10}$ O. Norniella, ${ }^{25}$ E. Nurse,${ }^{31}$ L. Oakes,${ }^{43}$ S. H. Oh, ${ }^{17}$ Y. D. Oh, ${ }^{28}$ I. Oksuzian, ${ }^{19}$ T. Okusawa,${ }^{42}$ R. Orava,${ }^{24}$ K. Osterberg,${ }^{24}$ S. Pagan Griso, ${ }^{44 b, 44 a}$ C. Pagliarone,${ }^{55 a}$ E. Palencia, ${ }^{18}$ V. Papadimitriou,${ }^{18}$ A. Papaikonomou, ${ }^{27}$ A. A. Paramonov, ${ }^{14}$ B. Parks,${ }^{40}$ S. Pashapour, ${ }^{34}$ J. Patrick, ${ }^{18}$ G. Pauletta, ${ }^{55 b, 55 a}$ M. Paulini, ${ }^{13}$ C. Paus, ${ }^{33}$ T. Peiffer, ${ }^{27}$ D. E. Pellett,${ }^{8}$ A. Penzo, ${ }^{55 a}$ T. J. Phillips, ${ }^{17}$ G. Piacentino, ${ }^{47 a}$ E. Pianori, ${ }^{46}$ L. Pinera, ${ }^{19}$ K. Pitts, ${ }^{25}$ C. Plager, ${ }^{9}$ L. Pondrom, ${ }^{60}$ O. Poukhov,${ }^{16, a}$ N. Pounder, ${ }^{43}$ F. Prakoshyn, ${ }^{16}$ A. Pronko, ${ }^{18}$ J. Proudfoot, ${ }^{2}$ F. Ptohos, ${ }^{18, j}$ E. Pueschel,${ }^{13}$ G. Punzi, ${ }^{47 b, 47 a}$ J. Pursley,${ }^{60}$ J. Rademacker, ${ }^{43, d}$ A. Rahaman, ${ }^{48}$

V. Ramakrishnan, ${ }^{60}$ N. Ranjan, ${ }^{49}$ I. Redondo, ${ }^{32}$ P. Renton, ${ }^{43}$ M. Renz, ${ }^{27}$ M. Rescigno, ${ }^{52 a}$ S. Richter, ${ }^{27}$ F. Rimondi, ${ }^{6 b, 6 a}$ L. Ristori, ${ }^{47 a}$ A. Robson, ${ }^{22}$ T. Rodrigo, ${ }^{12}$ T. Rodriguez, ${ }^{46}$ E. Rogers,${ }^{25}$ S. Rolli, ${ }^{57}$ R. Roser,${ }^{18}$ M. Rossi,${ }^{55 a}$ R. Rossin, ${ }^{11}$ P. Roy, ${ }^{34}$ A. Ruiz, ${ }^{12}$ J. Russ, ${ }^{13}$ V. Rusu, ${ }^{18}$ B. Rutherford, ${ }^{18}$ H. Saarikko, ${ }^{24}$ A. Safonov, ${ }^{54}$ W. K. Sakumoto, ${ }^{50}$ O. Saltó, L. Santi, ${ }^{55 b, 55 a}$ S. Sarkar, ${ }^{52 b, 52 a}$ L. Sartori, ${ }^{47 a}$ K. Sato, ${ }^{18}$ A. Savoy-Navarro, ${ }^{45}$ P. Schlabach, ${ }^{18}$ A. Schmidt,${ }^{27}$

E. E. Schmidt, ${ }^{18}$ M. A. Schmidt, ${ }^{14}$ M. P. Schmidt, ${ }^{61, a}$ M. Schmitt, ${ }^{39}$ T. Schwarz, ${ }^{8}$ L. Scodellaro, ${ }^{12}$ A. Scribano, ${ }^{47 c, 47 a}$ F. Scuri, ${ }^{47 a}$ A. Sedov, ${ }^{49}$ S. Seidel, ${ }^{38}$ Y. Seiya, ${ }^{42}$ A. Semenov ${ }^{16}$ L. Sexton-Kennedy, ${ }^{18}$ F. Sforza, ${ }^{47 b, 47 a}$ A. Sfyrla, ${ }^{25}$ S.Z. Shalhout, ${ }^{59}$ T. Shears,${ }^{30}$ P. F. Shepard, ${ }^{48}$ M. Shimojima, ${ }^{56, s}$ S. Shiraishi, ${ }^{14}$ M. Shochet, ${ }^{14}$ Y. Shon,${ }^{60}$ I. Shreyber, ${ }^{37}$ A. Simonenko, ${ }^{16}$ P. Sinervo, ${ }^{34}$ A. Sisakyan, ${ }^{16}$ A. J. Slaughter, ${ }^{18}$ J. Slaunwhite, ${ }^{40}$ K. Sliwa,${ }^{57}$ J. R. Smith, ${ }^{8}$ F. D. Snider, ${ }^{18}$ R. Snihur ${ }^{34}$ A. Soha,${ }^{8}$ S. Somalwar, ${ }^{53}$ V. Sorin, ${ }^{36}$ T. Spreitzer ${ }^{34}$ P. Squillacioti, ${ }^{47 c, 47 a}$ M. Stanitzki, ${ }^{61}$ R. St. Denis, ${ }^{22}$ B. Stelzer, ${ }^{34}$ O. Stelzer-Chilton, ${ }^{34}$ D. Stentz, ${ }^{39}$ J. Strologas, ${ }^{38}$ G. L. Strycker, ${ }^{35}$ J. S. Suh, ${ }^{28}$ A. Sukhanov, ${ }^{19}$ I. Suslov, ${ }^{16}$

T. Suzuki ${ }^{56}$ A. Taffard ${ }^{25, g}$ R. Takashima, ${ }^{41}$ Y. Takeuchi,${ }^{56}$ R. Tanaka,${ }^{41}$ M. Tecchio, ${ }^{35}$ P. K. Teng, ${ }^{1}$ K. Terashi, ${ }^{51}$

J. Thom, ${ }^{18, i}$ A. S. Thompson, ${ }^{22}$ G. A. Thompson, ${ }^{25}$ E. Thomson, ${ }^{46}$ P. Tipton, ${ }^{61}$ P. Ttito-Guzmán, ${ }^{32}$ S. Tkaczyk,${ }^{18}$ D. Toback,${ }^{54} \mathrm{~S}$. Tokar, ${ }^{15} \mathrm{~K}$. Tollefson, ${ }^{36} \mathrm{~T}$. Tomura ${ }^{56} \mathrm{D}$. Tonelli, ${ }^{18} \mathrm{~S}$. Torre,${ }^{20} \mathrm{D}$. Torretta, ${ }^{18} \mathrm{P}$. Totaro, ${ }^{55 \mathrm{~b}, 55 \mathrm{a}} \mathrm{S}$. Tourneur, ${ }^{45}$ M. Trovato, ${ }^{47 \mathrm{~d}, 47 \mathrm{a} a}$ S.-Y. Tsai, ${ }^{1}$ Y. Tu, ${ }^{46}$ N. Turini,${ }^{47 c, 47 a}$ F. Ukegawa, ${ }^{56}$ S. Vallecorsa ${ }^{21}$ N. van Remortel, ${ }^{24, \mathrm{c}}$ A. Varganov, ${ }^{35}$ E. Vataga, ${ }^{47 \mathrm{~d}, 47 \mathrm{a}}$ F. Vázquez, ${ }^{19,0} \mathrm{G}$. Velev,${ }^{18} \mathrm{C}$. Vellidis, ${ }^{3} \mathrm{M}$. Vidal,${ }^{32} \mathrm{R}$. Vidal,${ }^{18} \mathrm{I}$. Vila, ${ }^{12} \mathrm{R}$. Vilar,${ }^{12} \mathrm{~T}$. Vine, ${ }^{31} \mathrm{M}$. Vogel,${ }^{38}$

I. Volobouev, ${ }^{29, v}$ G. Volpi, ${ }^{47 b, 47 a}$ P. Wagner ${ }^{46}$ R. G. Wagner, ${ }^{2}$ R. L. Wagner, ${ }^{18}$ W. Wagner, ${ }^{27, y}$ J. Wagner-Kuhr, ${ }^{27}$

T. Wakisaka, ${ }^{42}$ R. Wallny, ${ }^{9}$ S. M. Wang, ${ }^{1}$ A. Warburton, ${ }^{34}$ D. Waters,${ }^{31}$ M. Weinberger, ${ }^{54}$ J. Weinelt, ${ }^{27}$ W. C. Wester III, ${ }^{18}$ B. Whitehouse, ${ }^{57}$ D. Whiteson, ${ }^{46,9}$ A. B. Wicklund, ${ }^{2}$ E. Wicklund,${ }^{18}$ S. Wilbur, ${ }^{14}$ G. Williams,${ }^{34}$ H. H. Williams, ${ }^{46}$ P. Wilson, ${ }^{18}$ B. L. Winer,${ }^{40}$ P. Wittich, ${ }^{18, i}$ S. Wolbers, ${ }^{18}$ C. Wolfe,${ }^{14}$ T. Wright,${ }^{35}$ X. Wu,${ }^{21}$ F. Würthwein,${ }^{10}$ S. Xie, ${ }^{33}$ A. Yagil, ${ }^{10}$ K. Yamamoto, ${ }^{42}$ J. Yamaoka, ${ }^{17}$ U. K. Yang, ${ }^{14, \mathrm{r}}$ Y. C. Yang, ${ }^{28}$ W. M. Yao, ${ }^{29}$ G. P. Yeh, ${ }^{18}$ K. Yi, ${ }^{18, p}$ J. Yoh, ${ }^{18}$ K. Yorita, ${ }^{58}$ T. Yoshida, ${ }^{42, \mathrm{~m}}$ G. B. Yu, ${ }^{50}$ I. Yu, ${ }^{28}$ S. S. Yu, ${ }^{18}$ J. C. Yun, ${ }^{18}$ L. Zanello, ${ }^{52 b, 52 a}$ A. Zanetti, ${ }^{55}$ X. Zhang, ${ }^{25}$ Y. Zheng, ${ }^{9, e}$ and S. Zucchelli ${ }^{6 b, 6 a}$

(CDF Collaboration)

\author{
${ }^{1}$ Institute of Physics, Academia Sinica, Taipei, Taiwan 11529, Republic of China \\ ${ }^{2}$ Argonne National Laboratory, Argonne, Illinois 60439, USA \\ ${ }^{3}$ University of Athens, 15771 Athens, Greece \\ ${ }^{4}$ Institut de Fisica d'Altes Energies, Universitat Autonoma de Barcelona, E-08193, Bellaterra (Barcelona), Spain \\ ${ }^{5}$ Baylor University, Waco, Texas 76798, USA \\ ${ }^{6 a}$ Istituto Nazionale di Fisica Nucleare Bologna, I-40127 Bologna, Italy \\ ${ }^{6 \mathrm{~b}}$ University of Bologna, I-40127 Bologna, Italy \\ ${ }^{7}$ Brandeis University, Waltham, Massachusetts 02254, USA \\ ${ }^{8}$ University of California, Davis, Davis, California 95616, USA \\ ${ }^{9}$ University of California, Los Angeles, Los Angeles, California 90024, USA \\ ${ }^{10}$ University of California, San Diego, La Jolla, California 92093, USA \\ ${ }^{11}$ University of California, Santa Barbara, Santa Barbara, California 93106, USA \\ ${ }^{12}$ Instituto de Fisica de Cantabria, CSIC-University of Cantabria, 39005 Santander, Spain \\ ${ }^{13}$ Carnegie Mellon University, Pittsburgh, Pennsylvania 15213, USA \\ ${ }^{14}$ Enrico Fermi Institute, University of Chicago, Chicago, Illinois 60637, USA \\ ${ }^{15}$ Comenius University, 84248 Bratislava, Slovakia; Institute of Experimental Physics, 04001 Kosice, Slovakia \\ ${ }^{16}$ Joint Institute for Nuclear Research, RU-141980 Dubna, Russia \\ ${ }^{17}$ Duke University, Durham, North Carolina 27708, USA \\ ${ }^{18}$ Fermi National Accelerator Laboratory, Batavia, Illinois 60510, USA \\ ${ }^{19}$ University of Florida, Gainesville, Florida 32611, USA \\ ${ }^{20}$ Laboratori Nazionali di Frascati, Istituto Nazionale di Fisica Nucleare, I-00044 Frascati, Italy \\ ${ }^{21}$ University of Geneva, CH-1211 Geneva 4, Switzerland \\ ${ }^{22}$ Glasgow University, Glasgow G12 8QQ, United Kingdom
}




\author{
${ }^{23}$ Harvard University, Cambridge, Massachusetts 02138, USA \\ ${ }^{24}$ Division of High Energy Physics, Department of Physics, University of Helsinki \\ and Helsinki Institute of Physics, FIN-00014, Helsinki, Finland \\ ${ }^{25}$ University of Illinois, Urbana, Illinois 61801, USA \\ ${ }^{26}$ The Johns Hopkins University, Baltimore, Maryland 21218, USA \\ ${ }^{27}$ Institut für Experimentelle Kernphysik, Universität Karlsruhe, 76128 Karlsruhe, Germany \\ ${ }^{28}$ Center for High Energy Physics: Kyungpook National University, Daegu 702-701, Korea; \\ Seoul National University, Seoul 151-742, Korea; Sungkyunkwan University, Suwon 440-746, Korea; \\ Korea Institute of Science and Technology Information, Daejeon, 305-806, Korea; \\ Chonnam National University, Gwangju,500-757, Korea; Chonbuk National University, Jeonju 561-756, Korea \\ ${ }^{29}$ Ernest Orlando Lawrence Berkeley National Laboratory, Berkeley, California 94720, USA \\ ${ }^{30}$ University of Liverpool, Liverpool L69 7ZE, United Kingdom \\ ${ }^{31}$ University College London, London WC1E 6BT, United Kingdom \\ ${ }^{32}$ Centro de Investigaciones Energeticas, Medioambientales y Tecnologicas, E-28040 Madrid, Spain \\ ${ }^{33}$ Massachusetts Institute of Technology, Cambridge, Massachusetts 02139, USA \\ ${ }^{34}$ Institute of Particle Physics: McGill University, Montréal, Québec, Canada H3A 2T8; \\ Simon Fraser University, Burnaby, British Columbia, Canada V5A 1S6; \\ University of Toronto, Toronto, Ontario, Canada M5S 1A7; and TRIUMF, Vancouver, British Columbia, Canada V6T $2 A 3$ \\ ${ }^{35}$ University of Michigan, Ann Arbor, Michigan 48109, USA \\ ${ }^{36}$ Michigan State University, East Lansing, Michigan 48824, USA \\ ${ }^{37}$ Institution for Theoretical and Experimental Physics, ITEP, Moscow 117259, Russia \\ ${ }^{38}$ University of New Mexico, Albuquerque, New Mexico 87131, USA \\ ${ }^{39}$ Northwestern University, Evanston, Illinois 60208, USA \\ ${ }^{40}$ The Ohio State University, Columbus, Ohio 43210, USA \\ ${ }^{41}$ Okayama University, Okayama 700-8530, Japan \\ ${ }^{42}$ Osaka City University, Osaka 588, Japan \\ ${ }^{43}$ University of Oxford, Oxford OX1 3RH, United Kingdom \\ ${ }^{44 \mathrm{a}}$ Istituto Nazionale di Fisica Nucleare, Sezione di Padova-Trento, I-35131 Padova, Italy \\ ${ }^{44 \mathrm{~b}}$ University of Padova, I-35131 Padova, Italy \\ ${ }^{45}$ LPNHE, Universite Pierre et Marie Curie/IN2P3-CNRS, UMR7585, Paris, F-75252 France \\ ${ }^{46}$ University of Pennsylvania, Philadelphia, Pennsylvania 19104, USA \\ ${ }^{47 a}$ Istituto Nazionale di Fisica Nucleare Pisa, I-56127 Pisa, Italy \\ ${ }^{47 \mathrm{~b}}$ University of Pisa, I-56127 Pisa, Italy \\ ${ }^{47 c}$ University of Siena, I-56127 Pisa, Italy \\ ${ }^{47 \mathrm{~d}}$ Scuola Normale Superiore, I-56127 Pisa, Italy \\ ${ }^{48}$ University of Pittsburgh, Pittsburgh, Pennsylvania 15260, USA \\ ${ }^{49}$ Purdue University, West Lafayette, Indiana 47907, USA
}

\footnotetext{
${ }^{\mathrm{a}}$ Deceased.

${ }^{\mathrm{b}}$ Visitor from University of Massachusetts Amherst, Amherst, MA 01003, USA.

${ }^{\mathrm{c}}$ Visitor from Universiteit Antwerpen, B-2610 Antwerp, Belgium.

${ }^{\mathrm{d}}$ Visitor from University of Bristol, Bristol BS8 1TL, United Kingdom.

${ }^{\mathrm{e}}$ Visitor from Chinese Academy of Sciences, Beijing 100864 , China.

${ }^{\mathrm{f}}$ Visitor from Istituto Nazionale di Fisica Nucleare, Sezione di Cagliari, 09042 Monserrato (Cagliari), Italy.

${ }^{\mathrm{g}}$ Visitor from University of California Irvine, Irvine, CA 92697, USA.

${ }^{\mathrm{h}}$ Visitor from University of California Santa Cruz, Santa Cruz, CA 95064, USA.

${ }^{\mathrm{i}}$ Visitor from Cornell University, Ithaca, NY 14853, USA.

${ }^{\mathrm{j}}$ Visitor from University of Cyprus, Nicosia CY-1678, Cyprus.

${ }^{\mathrm{k}}$ Visitor from University College Dublin, Dublin 4, Ireland.

${ }^{1}$ Visitor from University of Edinburgh, Edinburgh EH9 3JZ, United Kingdom.

${ }^{\mathrm{m}}$ Visitor from University of Fukui, Fukui City, Fukui Prefecture, Japan 910-0017.

${ }^{\mathrm{n}}$ Visitor from Kinki University, Higashi-Osaka City, Japan $577-8502$.
}

${ }^{\circ}$ Visitor from Universidad Iberoamericana, Mexico D.F., Mexico.

${ }^{\mathrm{p}}$ Visitor from University of Iowa, Iowa City, IA 52242, USA.

${ }^{\mathrm{q}}$ Visitor from Queen Mary, University of London, London, E1 4NS, United Kingdom.

${ }^{\mathrm{r}}$ Visitor from University of Manchester, Manchester M13 9PL, United Kingdom.

${ }^{\mathrm{s}}$ Visitor from Nagasaki Institute of Applied Science, Nagasaki, Japan.

${ }^{t}$ Visitor from University of Notre Dame, Notre Dame, IN 46556, USA.

${ }^{\mathrm{u}}$ Visitor from University de Oviedo, E-33007 Oviedo, Spain.

${ }^{\vee}$ Visitor from Texas Tech University, Lubbock, TX 79609 , USA.

${ }^{\text {w}}$ Visitor from IFIC (CSIC-Universitat de Valencia), 46071 Valencia, Spain.

${ }^{\mathrm{x}}$ Visitor from University of Virginia, Charlottesville, VA 22904, USA.

${ }^{\mathrm{y}}$ Visitor from Bergische Universität Wuppertal, 42097 Wuppertal, Germany.

${ }^{\mathrm{z}}$ On leave from J. Stefan Institute, Ljubljana, Slovenia. 


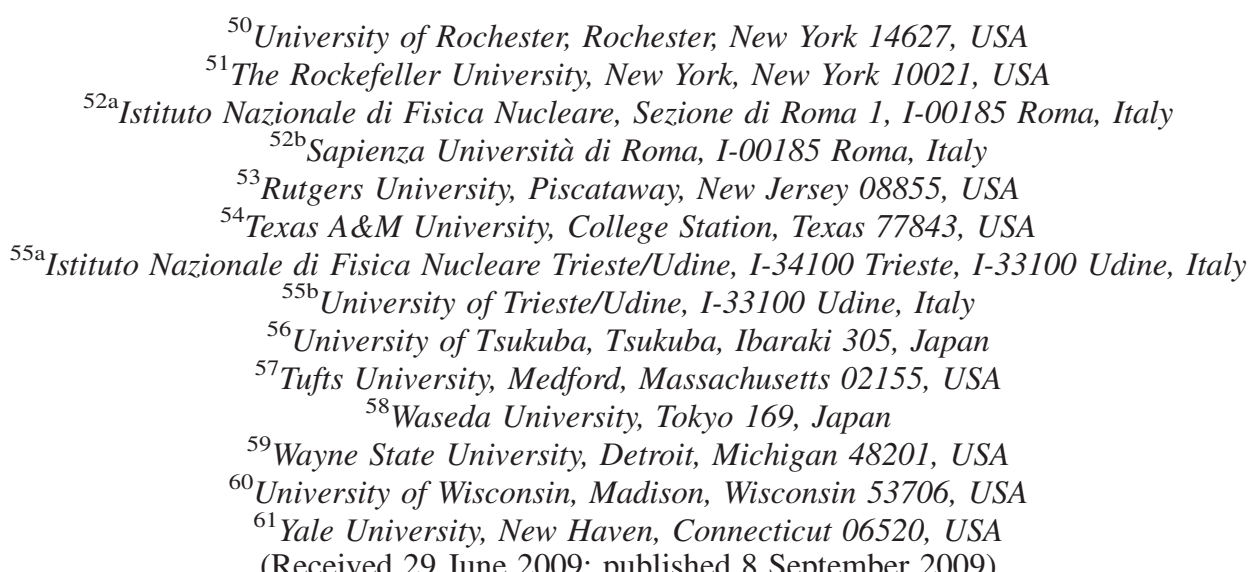

We present the first measurement of the mass of the top quark in a sample of $t \bar{t} \rightarrow \ell \bar{\nu} b \bar{b} q \bar{q}$ events (where $\ell=e, \mu$ ) selected by identifying jets containing a muon candidate from the semileptonic decay of heavy-flavor hadrons (soft muon $b$ tagging). The $p \bar{p}$ collision data used correspond to an integrated luminosity of $2 \mathrm{fb}^{-1}$ and were collected by the CDF II detector at the Fermilab Tevatron Collider. The measurement is based on a novel technique exploiting the invariant mass of a subset of the decay particles, specifically the lepton from the $W$ boson of the $t \rightarrow W b$ decay and the muon from a semileptonic $b$ decay. We fit template histograms, derived from simulation of $t \bar{t}$ events and a modeling of the background, to the mass distribution observed in the data and measure a top quark mass of $180.5 \pm 12.0$ (stat) \pm 3.6(syst) $\mathrm{GeV} / c^{2}$, consistent with the current world average value.

DOI: 10.1103/PhysRevD.80.051104

PACS numbers: $14.65 . \mathrm{Ha}, 12.15 . \mathrm{Ff}$

A massive top quark plays an important role in the standard model $(\mathrm{SM})$. The mass of the top quark $\left(m_{t}\right)$ enters electroweak precision observables as an input parameter via quantum effects, i.e., loop corrections, and its large numerical value gives rise to sizable corrections that behave as powers of $m_{t}$ [1]. For example, in the theoretical prediction of the $W$ boson mass $\left(m_{W}\right)$ within the SM, when these corrections are combined with the logarithmic dependence on the mass of the postulated Higgs boson $\left(m_{H}\right)$, a relationship emerges that provides a constraint on $m_{H}$ from experimental determinations of $m_{W}$ and $m_{t}$ [2]. Indeed, the strong dependence of the SM radiative corrections on $m_{t}$ made it possible to predict the value of $m_{t}$ [3] prior to its experimental determination $[4,5]$. Thus, a precision value of $m_{t}$ is crucial for constraining SM parameters, for high-sensitivity searches for effects of new physics, and for stringent consistency tests of models beyond the SM (e.g., supersymmetry). Furthermore, independent measurements of $m_{t}$ in all final states of $t \bar{t}$ decay provide an important consistency check of the top quark sector of the SM, and might reveal new physics with toplike signatures.

Significant progress has been made recently in reducing the uncertainty in measurements of $m_{t}$ and in devising alternative and independent techniques. The current best single measurement is determined by reconstructing the full decay chain and computing the invariant mass of the decay products in $t \bar{t} \rightarrow \ell \bar{\nu} b \bar{b} q \bar{q}$ events, and yields $m_{t}=$ $172.1 \pm 1.6 \mathrm{GeV} / c^{2}[6,7]$. However, this and all the most precise of the current techniques are limited by the common systematic uncertainty in the calorimeter jet energy calibration [jet energy scale (JES)]. To provide independent measurements, several techniques with minimal dependence on the JES have been proposed. For example, the flight distance of the $b$ hadron from the top decay can be used to infer the mass of the top quark [8], but this method also requires precision track reconstruction to determine the decay length. A proposal has been made [9] for exploiting the correlation between $m_{t}$ and the invariant mass of the system composed of a $J / \psi$ (from the decay of a $b$ hadron) and the lepton from the $W$ decay. The advantage is a stronger correlation of this system mass with $m_{t}$ than that of individual decay products of the top quark, and thus a better sensitivity to the top quark mass, but the overall branching fraction for this final state is only $\mathcal{O}\left(10^{-5}\right)$.

We present the first measurement of the mass of the top quark in a sample of $t \bar{t} \rightarrow \ell \bar{\nu} b \bar{b} q \bar{q}$ events (where $\ell=e, \mu$ ) selected by identifying $b$ jets with a candidate muon from semileptonic decay of heavy-flavor hadrons. We have developed a novel technique that exploits the invariant mass of the lepton from the $W$ boson of the $t \rightarrow W b$ decay, and the muon from a semileptonic $b$ decay. The selection method is complementary to that taking advantage of the long lifetime of $b$ hadrons through the presence of a decay vertex displaced from the primary interaction. Since only $\sim 50 \%$ of the sample of $t \bar{t}$ candidates with a semileptonic $b$ decay overlaps the top samples selected by the identification of a displaced vertex, and a still smaller fraction is in 
common with traditional samples that require all four jets for the mass reconstruction, our technique provides an essentially independent measurement of $m_{t}$ from these data. Moreover, our observable is largely independent of the JES, because the calorimeter information is used solely for the selection of event candidates, and therefore the result can add a significant amount of information when averaged with those from other measurements. Including sequential decays of charm, the branching fraction for $b \rightarrow$ $\mu \nu X \simeq 20 \%$ [2] is sizable and since this technique does not require precision secondary vertex reconstruction to suppress backgrounds, it could be an attractive option for the early phase of experiments at the Large Hadron Collider (LHC). Finally, the observable has a higher correlation to the top quark mass than the momentum of the lepton from the $W$ decay alone. A partial reduction in sensitivity will arise from $b-W$ mispairing, when the lepton from the $W$ decay and the muon from the $b$ semileptonic decay do not originate from the same top quark.

Top quarks are produced at the Tevatron protonantiproton collider predominantly in pairs of $t$ and $\bar{t}$, and are identified by the SM decay $t \rightarrow W b$, providing a final state that includes two $W$ bosons and two bottom quarks. $W$ 's are identified through their decay to leptons or quarks. Quarks hadronize and are observed as jets of charged and neutral particles. The CDF II detector is described in detail elsewhere [10]. The components relevant to this analysis include the central outer tracker (COT), the central electromagnetic and hadronic calorimeters, the central muon detectors and the luminosity counters. The data sample, produced in $p \bar{p}$ collisions at $\sqrt{s}=1.96 \mathrm{TeV}$ during run II of the Fermilab Tevatron, was collected between March 2002 and May 2007 and corresponds to an integrated luminosity of $2.0 \pm 0.1 \mathrm{fb}^{-1}$. We select events where one of the $W$ bosons decays to an isolated electron (muon) carrying large transverse energy $\left(E_{T}\right)$ [momentum $\left(p_{T}\right)$ ] [11] with respect to the beam line, plus a neutrino. We refer to these high- $p_{T}$ electrons or muons as primary leptons (PLs). The neutrino escapes the detector causing an imbalance of the total transverse energy vector, referred to as missing $E_{T}\left(\mathscr{E}_{T}\right)$. The other $W$ boson in the event decays hadronically to a pair of quarks. We take advantage of the semileptonic decay of $B$ hadrons by searching for muons within final-state jets [soft-lepton tagging (SLT)], in order to identify those jets that result from hadronization of the bottom quarks.

The event selection starts with an inclusive lepton trigger requiring an electron (muon) with $E_{T}>18 \mathrm{GeV}\left(p_{T}>\right.$ $18 \mathrm{GeV} / c)$. Further selection requires that candidate electron (muon) PLs are isolated and have $E_{T}>20 \mathrm{GeV}$ $\left(p_{T}>20 \mathrm{GeV} / c\right)$ and $|\eta|<1.1$. We define an isolation parameter $I$ as the calorimeter transverse energy in a cone of opening $\Delta R \equiv \sqrt{(\Delta \eta)^{2}+(\Delta \phi)^{2}}=0.4$ around the lepton (not including the lepton energy itself) divided by the electron $E_{T}$ or muon $p_{T}$. We select isolated electrons (muons) by requiring $I<0.1$. The event must have $\mathbb{E}_{T}>$ $30 \mathrm{GeV}$, consistent with the presence of a neutrino from the $W$ boson decay. Jets are identified using a fixed-cone algorithm with a cone opening of $\Delta R=0.4$ and are constrained to originate from the $p \bar{p}$ collision vertex. Muons inside jets are identified by matching the tracks of the jet, as measured in the COT, with track segments in the muon detectors. Such a muon with $p_{T}>3 \mathrm{GeV} / c$ and within $\Delta R<0.6$ of a jet axis is called an SLT $\mu$. The probability of misidentifying a hadron as an SLT $\mu$, denoted as the SLT $\mu$ mistag probability, is measured using a data sample of pions, kaons and protons from $D^{*}$ and $\Lambda^{0}$ decays. A Monte Carlo (MC) simulation of $W+$ jets events, whose details are given below, is used to model the $\pi, K$ and $p$ admixture in light-quark jets [12]. The SLT $\mu$ mistag probability is parametrized as a function of the track $p_{T}$ and $\eta$, and is shown to describe within $\pm 5 \%$ the number of false SLT $\mu$ tags in candidate light-flavor jets of QCD multijet and $\gamma+$ jet events.

To reduce background from dimuon resonances and double-semileptonic $B$ hadron decays, we remove events in which the PL muon and $\operatorname{SLT} \mu$ are oppositely charged and have an invariant mass consistent with a $Z, Y$ or, irrespectively of the PL flavor, less than $5 \mathrm{GeV} / c^{2}$. We further reject events as candidate radiative Drell-Yan and $Z$ bosons if the tagged jet has an electromagnetic energy fraction above 0.8 and only one track with $p_{T}>$ $1.0 \mathrm{GeV} / c$ within a cone of $\Delta R=0.4$ about the jet axis. The jet energies are corrected to account for variations of the detector response in $\eta$ and time, calorimeter gain drifts, nonlinearity of calorimeter energy response, multiple $p \bar{p}$ interactions in an event and for energy loss in uninstrumented regions [13]. Finally, the sample is partitioned according to the number of jets with $E_{T}>20 \mathrm{GeV}$ and $|\eta|<2.0$ in the event, and at least one jet is required to contain an $\operatorname{SLT} \mu$ (defining the SLT $\mu$-tagged $W+n$ jets sample). The subset of $W$ plus at least 3 jets is the $t \bar{t}$ candidate sample, and to reduce background from QCD production of $W$ boson with multiple jets, we additionally require the total transverse scalar energy in the event $\left(H_{T}\right.$ [14]) to be greater than $200 \mathrm{GeV}$.

Standard model processes that result in the same signature as the $t \bar{t}$ signal are backgrounds to this measurement. There are three dominant backgrounds: the largest one is mistags of $W+$ light-flavor events, and a smaller contribution is due to the $W$ boson in association with heavyflavor jets $(W b \bar{b}, W c \bar{c}, W c)$. Events without $W$ bosons that pass the event selection are typically QCD multijet events where one jet is reconstructed as a high- $p_{T}$ lepton, mismeasured jet energies produce apparent $\mathscr{E}_{T}$ and an additional jet contains an SLT $\mu$. A fraction of these events is from $b \bar{b}$ and $c \bar{c}$, where the candidate PL may result from a semileptonic decay of one of the fragmenting heavy quark and the SLT $\mu$ from a semileptonic decay of the other. Other minor backgrounds that can mimic a $W$ boson and 
an $\operatorname{SLT} \mu$ signature include diboson $(W W, Z Z, W Z)$, Drell-Yan $\rightarrow \tau \tau$, single top quark, and residual Drell-Yan $\rightarrow \mu \mu$ events not removed by the dimuon resonance removal. The composition of the data sample used in this analysis has been studied extensively in [12], where we have measured the production cross section for $p \bar{p} \rightarrow t \bar{t} X$, and is summarized in Table I. The $W+$ jets, QCD multijet and Drell-Yan background are determined using the data, while the remaining backgrounds are estimated from MC simulations. The $W+1,2$ jets samples contain little $t \bar{t}$ events and have a composition similar to the background of the $t \bar{t}$ candidate sample. The simulation of $t \bar{t}$ events is performed using PYTHIA [15] and HERWIG [16]. The generators are used with the CTEQ5L [17] parton distribution functions (PDFs). Modeling of $b$ and $c$ hadron decay is provided by EVTGEN [18]. Modeling of $W+$ jets production is performed using ALPGEN [19], coupled with PYTHIA for the shower evolution and EVTGEN for the heavy-flavor hadron decays. Diboson production $(W W, Z Z, W Z)$ and Drell-Yan $\rightarrow \tau \tau$ are determined using PYTHIA. Drell-Yan $\rightarrow \mu \mu+$ jets events are modeled using ALPGEN while single top production is modeled with MADEVENT [20], both with PYTHIA showering. The CDF II detector simulation models the response of the detector to particles produced in $p \bar{p}$ collisions. The detector geometry used in the simulation is the same as that used for reconstruction of the collision data. Details of the CDF II simulation, based on the GEANT3 package, can be found in [21].

We compute the invariant mass $\left(M_{\ell \mu}\right)$ between the PL and the SLT $\mu$ in the $t \bar{t}$ candidates sample. In rare cases where there is more than one SLT $\mu$ tag in the same jet, or more than one SLT $\mu$ tagged jet in the same event, we use the SLT $\mu$ candidate that has the best match between the COT track and the track segment in the muon detectors. No attempt is made to choose the correct pairing from the decay chain of the two top quarks. The electric charge of the SLT $\mu$, for instance, is not an effective flavor selector due to abundant sequential $b \rightarrow c \rightarrow \mu$ decays. When the

TABLE I. Composition of the SLT $\mu$-tagged $W+n$ jets candidate sample [12]. The $H_{T}>200 \mathrm{GeV}$ requirement is released for events with fewer than 3 jets.

\begin{tabular}{lccr}
\hline \hline Source & $W+1$ jet & $W+2$ jet & $W+\geq 3$ jets \\
\hline$W+$ light flavor & $622 \pm 31$ & $226 \pm 12$ & $52.3 \pm 2.6$ \\
$W+$ heavy flavor & $145 \pm 55$ & $67 \pm 25$ & $14.3 \pm 5.4$ \\
QCD multijet & $92 \pm 16$ & $45 \pm 10$ & $6.9 \pm 1.5$ \\
$W W+W Z+Z Z$ & $3.8 \pm 0.4$ & $7.0 \pm 0.7$ & $1.9 \pm 0.3$ \\
Drell-Yan $\rightarrow \tau \tau$ & $2.6 \pm 0.6$ & $1.5 \pm 0.4$ & $0.6 \pm 0.3$ \\
Drell-Yan $\rightarrow \mu \mu$ & $6.0 \pm 1.2$ & $4.1 \pm 0.9$ & $0.8 \pm 0.5$ \\
Single top & $4.4 \pm 0.4$ & $9.0 \pm 0.7$ & $2.7 \pm 0.2$ \\
Total background & $876 \pm 54$ & $359 \pm 24$ & $79.5 \pm 5.3$ \\
$t \bar{t}\left(\sigma_{t \bar{t}}=9.1 \mathrm{pb}\right)$ & $3.5 \pm 0.2$ & $31.8 \pm 1.0$ & $168.5 \pm 5.3$ \\
Data & 892 & 384 & 248 \\
\hline \hline
\end{tabular}

wrong pairing is chosen, there is still sensitivity to the top quark mass due to the boost of the SLT $\mu$ and the PL. The distribution of $M_{\ell \mu}$ is given by the contribution of $t \bar{t}$ and background events. For the background, the $M_{\ell \mu}$ distribution of QCD multijet events is derived from the data themselves in the kinematic region of $I>0.15, \mathbb{E}_{T}>$ $30 \mathrm{GeV}$, topologically close to the signal region, while for other background sources we use MC simulation. We check the background model in $W+1,2$ jet SLT $\mu$-tagged data events, a sample with a similar composition as the background to $t \bar{t}$ candidates. We find the predicted and observed distributions of $M_{\ell \mu}$ (Fig. 1) to be in agreement with a $p$ value of $55 \%$, as given by the KolmogorovSmirnov test.

We construct a set of template histograms of the $M_{\ell \mu}$ distribution using the background model and a simulation of $t \bar{t}$ events. The $t \bar{t}$ samples are generated with different top quark mass values in the range $150-195 \mathrm{GeV} / c^{2}$, incrementing by steps of up to $0.5 \mathrm{GeV} / c^{2}$, and the full $M_{\ell \mu}$ spectra are determined by adding the signal and expected background histograms in the ratio shown in Table I. Figure 2 shows the mean value of the $M_{\ell \mu}$ distributions versus the input top quark mass, indicating a linear relationship between the two quantities. Also shown is $\left\langle M_{\ell \mu}\right\rangle=35.6 \pm 1.1$ (stat) $\mathrm{GeV} / c^{2}$, measured in the data. We perform a binned-likelihood fit to the $M_{\ell \mu}$ histogram of the data, in 20 bins between 4 and $100 \mathrm{GeV} / c^{2}$, with the binning and range chosen a priori appropriately to the size of the data sample. The likelihood is defined as

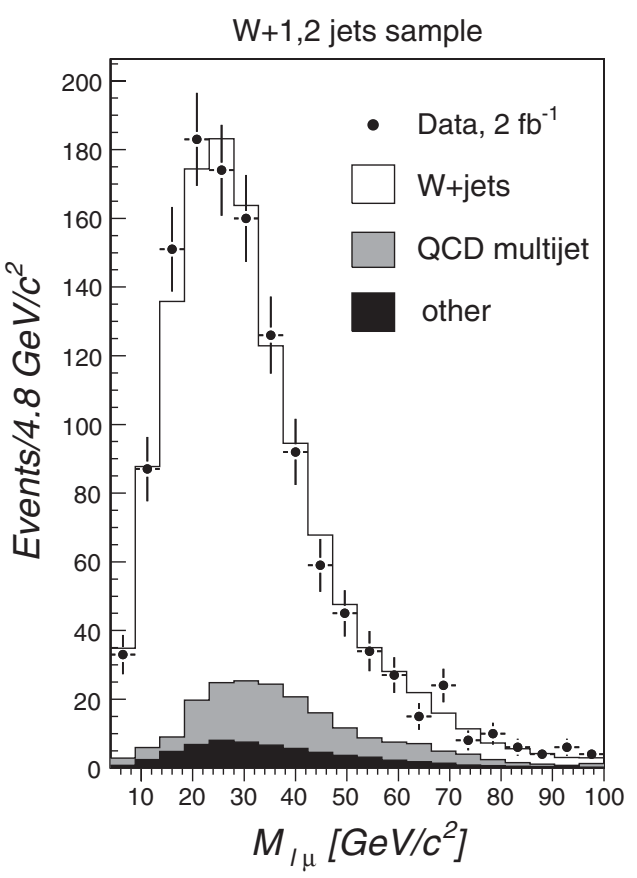

FIG. 1. The predicted and observed $M_{\ell \mu}$ distributions in the sample of $W+1,2$ jet SLT $\mu$-tagged events. The predicted distributions are stacked. 


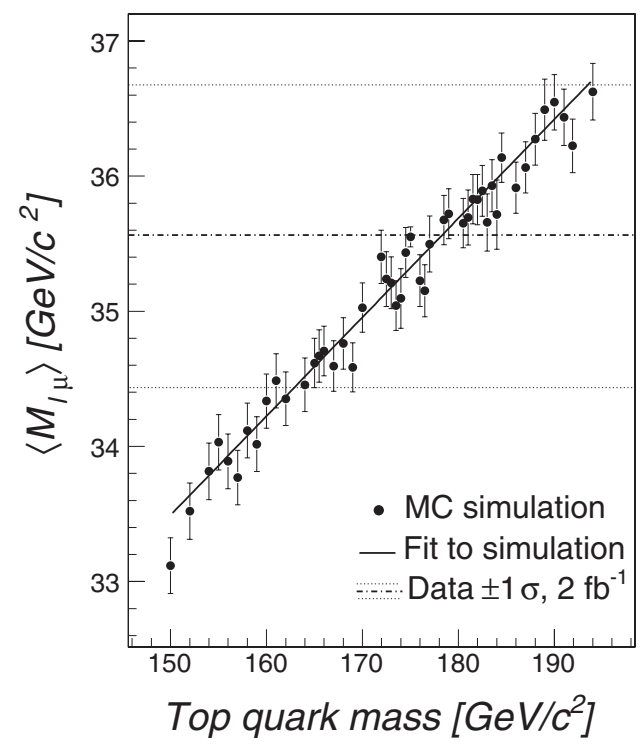

FIG. 2. The correlation between the mean value of the $M_{\ell \mu}$ histograms from simulated $t \bar{t}$ and background samples, and the input $m_{t}$. The continuous line shows a linear fit to the points.

$$
-\ln L\left(m_{t}\right)=-\sum_{i=1}^{\mathrm{N}_{\mathrm{bins}}} n_{i}^{\text {data }} \ln \left[\frac{n_{i}^{\mathrm{TP}}\left(m_{t}\right)}{n_{\mathrm{tot}}^{\mathrm{TP}}}\right],
$$

where $n_{i}^{\text {data }}$ and $n_{i}^{\mathrm{TP}}\left(m_{t}\right)$ are the number of entries in each $i$ bin of the data and template histograms, respectively, the total number of entries is $n_{\mathrm{tot}}^{\mathrm{TP}}=n_{\mathrm{tot}}^{\mathrm{data}}$, and $n_{i}^{\mathrm{TP}}\left(m_{t}\right) / n_{\mathrm{tot}}^{\mathrm{TP}} \equiv$ $\mathcal{P}_{i}\left(m_{t}\right)$ is the probability of the $i$ th bin, normalized such that $\sum_{i} \mathcal{P}_{i}=1$. The background normalization is fixed and its value is varied in the evaluation of the systematic uncertainty. A parabolic function is fit to the values of $\ln L\left(m_{t}\right)$ derived from each mass template, and the measured top quark mass is determined from the minimum of the likelihood function, while the statistical uncertainty is given by the range corresponding to an increase in the $-\ln L$ of 0.5 units above the minimum. For each mass point within the full mass range, we generate 5000 pseudoexperiments with the same sample size as that of the data and verify that the fitting procedure is unbiased and that the statistical uncertainty returned by the fits represents the $68 \%$ confidence level. From $248 t \bar{t}$ candidate events, we measure:

$$
m_{t}=180.5 \pm 12.0(\text { stat }) \pm 3.6(\text { syst }) \mathrm{GeV} / c^{2} .
$$

Figure 3 shows the $M_{\ell \mu}$ distribution of the data, the background, and the templates corresponding to the best fit and the statistical uncertainty.

The sources of systematic uncertainty that affect the measured value of the top quark mass are summarized in Table II. The limited size of the $t \bar{t}$ samples simulated with different values of $m_{t}$, input to the fitting procedure, yields an uncertainty of $\pm 0.3 \mathrm{GeV} / c^{2}$. Several components enter the uncertainty on the modeling of the background. The uncertainty on the $W+$ heavy- and light-flavor normalizations yields an uncertainty of $\pm 0.5 \mathrm{GeV} / c^{2}$. The uncer-

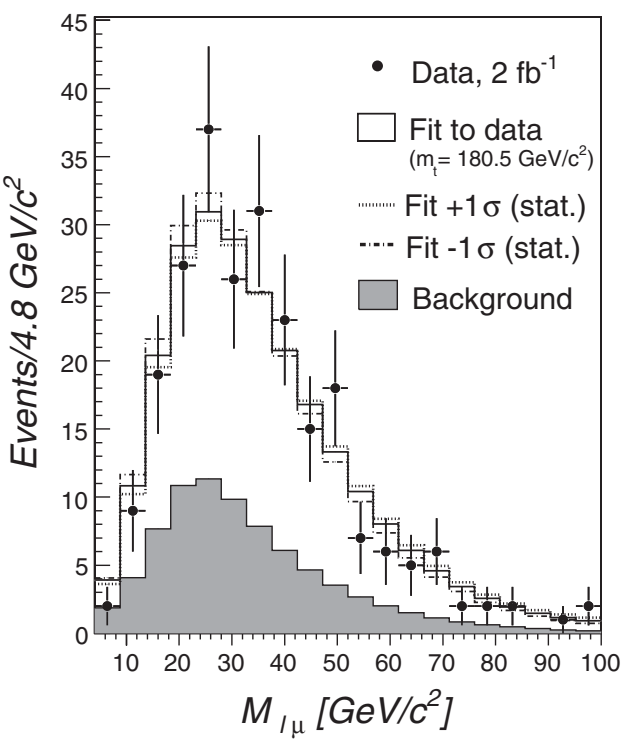

FIG. 3. The distribution of invariant mass $M_{\ell \mu}$ of the lepton from the $W$ decay and the SLT $\mu$, from a sample of 248 candidate $t \bar{t}$ events with 79.5 background.

tainty on the shape of the $W+$ jets histogram is evaluated by varying the distribution, to within the statistical accuracy associated with the comparison in the $W+1,2$ jets sample between the data and the background model, and yields an uncertainty of $\pm 1.4 \mathrm{GeV} / c^{2}$. The normalization of the QCD multijet background contributes $\pm 0.8 \mathrm{GeV} / c^{2}$. The shape of the QCD multijet distribution accounts for $\pm 0.6 \mathrm{GeV} / c^{2}$, as determined by replacing the nominal sample with dijet enriched data selected by $I<$ 0.1 and $\mathbb{E}_{T}<15 \mathrm{GeV}$, and by varying the distribution according to its statistical uncertainty. The shift on the measured top quark mass due to the uncertainties on the remaining backgrounds is negligible. The total uncertainty from background modeling is $\pm 1.9 \mathrm{GeV} / c^{2}$.

Monte Carlo modeling of the signal $M_{\ell \mu}$ distributions includes effects of PDFs, initial-state radiation (ISR), finalstate radiation (FSR), and JES. The uncertainty due to the MC modeling of $t \bar{t}$ production and decay, including $b$ fragmentation, is determined by comparing the simulation

TABLE II. Summary of systematic uncertainties.

\begin{tabular}{lc}
\hline \hline Source & $\Delta m_{\mathrm{t}}\left[\mathrm{GeV} / c^{2}\right]$ \\
\hline MC $t \bar{t}$ samples statistics & \pm 0.3 \\
Background & \pm 1.9 \\
$t \bar{t}$ production and decay model & \pm 2.1 \\
Parton distribution functions & \pm 1.0 \\
Initial- and final-state radiation & \pm 1.3 \\
Jet energy scale & \pm 0.3 \\
PL energy/momentum scale & \pm 0.9 \\
SLT $\mu$ momentum & \pm 0.9 \\
Pileup & \pm 0.5 \\
Total & \pm 3.6 \\
\hline \hline
\end{tabular}


using PYTHIA with that using HERWIG and gives $\Delta m_{t}=$ $\pm 2.1 \mathrm{GeV} / c^{2}$. The PDF uncertainty is evaluated by adding in quadrature the contribution of four effects: variations of the PDFs according to the 20 CTEQ eigenvectors [22], the difference between the standard $t \bar{t}$ simulation using the CTEQ5L PDF and one derived using MRST98 [23] in the default configuration or with two alternative choices for $\alpha_{s}$, and the variation of the contribution of gluon fusion in $t \bar{t}$ production between $5 \%$ and $20 \%$. The overall estimated uncertainty from PDF is $\pm 1.0 \mathrm{GeV} / c^{2}$. We vary both ISR and FSR simultaneously in the $t \bar{t}$ Monte Carlo simulation, within constraints set by studies of radiation in Drell-Yan events in the data, and assign a systematic uncertainty on $m_{t}$ of $\pm 1.3 \mathrm{GeV} / c^{2}$.

The jet reconstruction is used in this analysis only for the selection of event candidates and therefore the uncertainty on the calibration of the jet energies enters the measurement solely through the event selection, via the jet counting and the $\mathbb{E}_{T}$ requirement. The uncertainty due to the JES is measured by shifting the energies of the jets in the $t \bar{t} \mathrm{MC}$ simulation by $\pm 1 \sigma$ of the JES [13] and results in $\Delta m_{t}=$ $\pm 0.3 \mathrm{GeV} / c^{2}$. The uncertainty of $\pm 1 \%$ on the difference between data and simulation of the PL energy and momentum scales gives an uncertainty of $\pm 0.9 \mathrm{GeV} / c^{2}$. The differences in the data versus simulation for the $\operatorname{SLT} \mu$ $p_{T}$ spectrum depend on the $b$-quark fragmentation modeling and the momentum calibration. In addition to the different fragmentation models in HERWIG versus PYTHIA, we consider comparisons of the data with MC simulation of the muon $p_{T}$ spectra in $B \rightarrow \mu^{-} D^{0} X$ [24] and $b \bar{b} \rightarrow$ $\mu \mu X$ [25] which indicate an uncertainty on the muon $p_{T}$ of $\sim \pm 0.8 \%$, corresponding to $\Delta m_{t}= \pm 0.9 \mathrm{GeV} / c^{2}$. The uncertainty on the $p_{T}$ dependence of the SLT $\mu$ tagging efficiency yields a shift on the top quark mass of $\pm 0.2 \mathrm{GeV} / c^{2}$. Finally, a source of systematic uncertainty is due to the modeling of pileup events from multiple $p \bar{p}$ interactions and it is estimated to affect the measured mass by $\leq \pm 0.5 \mathrm{GeV} / c^{2}$.

In summary, we have performed the first measurement of the top quark mass in a sample of $t \bar{t} \rightarrow \ell \bar{\nu} b \bar{b} q \bar{q}$ events selected by identifying $b$ jets with a muon candidate from the semileptonic decay of heavy-flavor hadrons. The result, $m_{t}=180.5 \pm 12.0$ (stat) \pm 3.6 (syst) $\mathrm{GeV} / c^{2}$, is in agreement with the current world average value of $173.1 \pm$ $1.3 \mathrm{GeV} / c^{2}$ [6], providing a consistency check of the top quark sector with soft muon $b$-tagged events. Our measurement technique exploits the correlation between the parent top quark mass and the invariant mass of the system composed of the lepton from the $W$ decay and the muon from the semileptonic $B$ decay. The uncertainty at present is dominated by the statistical component. The method has a minimal dependence on the jet energy calibration, making it suitable for averaging the result with those from other techniques, and its dominant systematic uncertainties are likely reducible, e.g., by improving the calibration of the leptons' $p_{T}$ to better than $1 \%$ with $J / \psi, Y$ and $Z$ resonances, by using improved tuning for the MC modeling of $t \bar{t}$ production and decay, and with high statistics data samples for the background model.

We thank the Fermilab staff and the technical staffs of the participating institutions for their vital contributions. This work was supported by the U.S. Department of Energy and National Science Foundation; the Italian Istituto Nazionale di Fisica Nucleare; the Ministry of Education, Culture, Sports, Science and Technology of Japan; the Natural Sciences and Engineering Research Council of Canada; the National Science Council of the Republic of China; the Swiss National Science Foundation; the A.P. Sloan Foundation; the Bundesministerium für Bildung und Forschung, Germany; the Korean Science and Engineering Foundation and the Korean Research Foundation; the Science and Technology Facilities Council and the Royal Society, United Kingdom; the Institut National de Physique Nucleaire et Physique des Particules/CNRS; the Russian Foundation for Basic Research; the Ministerio de Ciencia e Innovación, and Programa Consolider-Ingenio 2010, Spain; the Slovak R\&D Agency; and the Academy of Finland.
[1] M. Veltman, Nucl. Phys. B123, 89 (1977).

[2] For a recent review, see C. Amsler et al. (Particle Data Group), Phys. Lett. B 667, 1 (2008).

[3] For example, see J. Ellis, G. Fogli, and E. Lisi, Phys. Lett. B 292, 427 (1992).

[4] S. Abachi et al., Phys. Rev. Lett. 79, 1197 (1997); B. Abbott et al., Phys. Rev. Lett. 80, 2063 (1998); Phys. Rev. D 58, 052001 (1998); 60, 052001 (1999).

[5] F. Abe et al., Phys. Rev. Lett. 80, 2767 (1998); 80, 2779 (1998); 82, 271 (1999).
[6] Tevatron Electroweak Working Group, arXiv:0903.2503v1.

[7] T. Aaltonen et al. (CDF Collaboration), Phys. Rev. D 79, 072001 (2009).

[8] A. Abulencia et al. (CDF Collaboration), Phys. Rev. D 75, 071102(R) (2007).

[9] CMS Letter of Intent, Report No. CERN/LHCC 92-3, 1992.

[10] The CDF II Detector Technical Design Report, Report No. Fermilab-Pub-96/390-E; D. Acosta et al. (CDF 
Collaboration), Phys. Rev. D 71, 032001 (2005).

[11] We use a $(z, \phi, \theta)$ coordinate system where the $z$ axis is in the direction of the proton beam and $\phi$ and $\theta$ are the azimuthal and polar angles, respectively. The pseudorapidity is $\eta \equiv-\ln \left(\tan \frac{\theta}{2}\right)$. The transverse energy and momentum are $E_{T} \equiv E \sin \theta$ and $p_{T} \equiv p \sin \theta$, respectively, where $E$ and $p$ are energy and momentum, respectively. The missing transverse energy is $\mathbb{E}_{T} \equiv\left|-\sum_{i} E_{T}^{i} \hat{n}_{i}\right|$, where $E_{T}^{i}$ is the magnitude of the transverse energy contained in each calorimeter tower $i$ in the region $|\eta|<3.6$ and $\hat{n}_{i}$ is the direction unit vector of the tower in the plane transverse to the beam direction.

[12] T. Aaltonen et al. (CDF Collaboration), Phys. Rev. D 79, 052007 (2009).

[13] A. Bhatti et al., Nucl. Instrum. Methods Phys. Res., Sect. A 566, 375 (2006).

[14] The $H_{T}$ is defined as the scalar sum of the electron (muon) $E_{T}\left(p_{T}\right), E_{T}$ and jet $E_{T}$ for jets with $E_{T}>8 \mathrm{GeV}$ and $|\eta|<2.4$.

[15] T. Sjostrand et al., Comput. Phys. Commun. 135, 238
(2001).

[16] G. Corcella et al., J. High Energy Phys. 01 (2001) 010.

[17] H. L. Lai et al., Eur. Phys. J. C 12, 375 (2000).

[18] D. Lange, Nucl. Instrum. Methods Phys. Res., Sect. A 462, 152 (2001).

[19] M. L. Mangano et al., J. High Energy Phys. 07 (2003) 001.

[20] F. Maltoni and T. Stelzer, J. High Energy Phys. 02 (2003) 027.

[21] E. Gerchtein and M. Paulini, in Proceedings of the 2003 Conference on Computing in High Energy and Nuclear Physics, econf C0303241, TUMT005 (2003).

[22] H. L. Lai et al., Eur. Phys. J. C 12, 375 (2000).

[23] A. D. Martin, R. G. Roberts, W. J. Stirling, and R. S. Thorne, Eur. Phys. J. C 4, 463 (1998).

[24] T. Aaltonen et al. (CDF Collaboration), Phys. Rev. D 79, 092003 (2009); J. A. Kraus, Ph.D. thesis, University of Illinois, 2006.

[25] T. Aaltonen et al. (CDF Collaboration), Phys. Rev. D 77, 072004 (2008). 